\title{
Pauci-immune glomerulonephritis with
} ANCA

INSERM

\section{Source}

INSERM. (1999). Orphanet: an online rare disease and orphan drug data base. Pauciimmune glomerulonephritis with ANCA. ORPHA:97563

Pauci-immune glomerulonephritis (GN) with antineutrophil cytoplasmic antibodies (ANCA) is a form of rapidly progressive GN comprising about $90 \%$ of pauci-immune glomerulonephritis (see this term), and associated with the presence of circulating ANCA (mostly directed against proteinase-3 (PR3) and myeloperoxidase (MPO)). Patients usually present with hematuria and rapidly declining renal function, often leading to dialysis within weeks without treatment. Cutaneous, pulmonary, musculoskeletal and nervous involvement may be observed in case of systemic disease, and the correlation between ANCA titer and disease activity has been demonstrated. 